\title{
Materia de teoría. Conferencias públicas y recepción de la ciencia en Argentina (1800-1930)
}

\author{
MIGUEL DE ASÚA Universidad Nacional de San Martín - CONICET, Argentina / mdeasua@yahoo.com
}

\section{Resumen}

En Argentina, un espacio científico ambiguamente periférico, algunas teorías de las ciencias exactas y naturales fueron recibidas de manera simultánea y (en parte) contradictoria por un micro-universo de expertos con acceso a los medios especializados y por el público general a través de conferencias públicas o textos con algún tipo de intención o aura estética. Es el caso de la teoría copernicana, de la evolución de Darwin y de la relatividad. Uno de estos formatos de difusión de teorías fueron los ejercicios literarios públicos emprendidos por actores culturales prominentes. Estos intérpretes buscaron legitimar intereses particulares, proyectos políticos o sistemas de ideas articulando el prestigio y autoridad social de la ciencia con el despliegue de recursos retóricos a efectos de comunicar más efectivamente su mensaje. En este trabajo reviso, desde esta perspectiva y con el trasfondo de las respectivas nubes contextuales, tres conferencias públicas históricamente significativas en las que tuvieron un papel relevante cada una de las teorías señaladas: la «Prolusión académica» de Pedro Cerviño (enero 1806), el discurso de Sarmiento en el «Funeral cívico» de Darwin (30 de mayo de 1882) y la conferencia de Lugones «El tamaño del espacio» (14 agosto de 1920).

Palabras clave: popularización de la ciencia / teoría de la evolución en Argentina / teoría de la relatividad en Argentina / Domingo F. Sarmiento / Leopoldo Lugones

\section{A Matter of Theory. Public Lectures and the Reception of Science in Argentina (1800-1930). Abstract}

In Argentina, an ambiguously peripheral space from the point of view of scientific culture, the theories of the natural and exact sciences were received simultaneously (and in part contradictorily) by a micro-universe of experts with access to specialized publications and by the general public through public lectures or texts with some kind of didactic or esthetical intention. This is the case of Copernican theory, Darwin's evolutionary theory and Einstein's theory of relativity. One of the formats through which these theories reached a wide audience were public lectures pronounced by prominent cultural actors. These interpreters sought to legitimatize personal interests, political programs or ideologies articulating the prestige and social authority of science with a deployment of rhetorical resources. In this paper I discuss three historically significant cases, in each of which the above mentioned theories played a prominent role: Pedro Cerviño's «Academic Exordium» (January 1806), Sarmiento's lecture on occasion of Darwin's «Civic Funeral» (20 May 1882) and Lugones' «The Size of Space» (14 August 1920).

Key words: popularization of science in Argentina / evolution theory in Argentina / relativity in Argentina / Domingo F. Sarmiento / Leopoldo Lugones

Fecha de recepción: 6/12/2017; fecha de aceptación: 1/3/2018

Para citar este artículo: De Asúa, Miguel (2018). «Materia de teoría. Conferencias públicas y recepción de la ciencia en Argentina (1800-1930)». El taco en la brea 7 (diciembre-mayo), 92-103 Santa Fe, Argentina: UNL. DOI: https://doi.org/10.14409/tb.voi7.7357 
Análogamente a la «teoría de la recepción» en estudios literarios, en historia de la ciencia tenemos su inversa simétrica, la «recepción de teorías». Es significativo que a partir de 1969 Hans Robert Jauss, el creador de la teoría de la recepción en literatura, haya sugerido en varias oportunidades que la formulación de su propio programa disciplinar constituía un cambio de paradigma, en el sentido original que el historiador y filósofo de la ciencia Thomas Kuhn le había otorgado a esta noción hacía entonces pocos años, en 1962 (Jauss; cf. Nauta:207; Kuhn). Jauss definía tres paradigmas en los métodos de crítica histórico-literaria previos a la aparición del suyo. Los inconvenientes del uso de la noción de Kuhn en este contexto han sido señalados por Robert Holub (5-6). Ciertamente, en las últimas décadas del siglo XX hubo un uso y abuso de la noción de paradigma (Asúa 2004a). En todo caso, si menciono este episodio es sólo porque llama la atención, aunque más no sea indirectamente, sobre el vago aire de familia entre las nociones de recepción de texto literario y recepción de teoría científica: ambas se ocupan de lo que podemos llamar el «polo distal» del proceso de transmisión de amplios sistemas de significación. Pero ir más allá en la búsqueda de simetrías entre las belles lettres (porque de eso trataba la teoría de la recepción) y las teorías de la ciencia es condenarse a una analogía vacía.

Otra cosa es la investigación del vehículo discursivo de teorías científicas. En una revisión aparecida en Isis hace seis años, sobre qué puede aprender la historia de la ciencia de los estudios literarios, Henry S. Turner discutió en extenso la transferencia metodológica de la noción de «forma». Un mínimo de conciencia lingüística indica que la consideración del tipo de texto a través del cual una teoría científica es presentada o divulgada puede contribuir a entender el proceso de su recepción, sobre todo en sus aspectos de metabolización cultural. En otras ocasiones hablé de «recepción literaria» de una teoría, pero a la distancia veo que esa expresión es cuestionable, toda vez que presupone el carácter estanco de un discurso «literario», que es justamente lo que no se advierte en los casos históricos, que más bien operan dentro de una ambigüedad genérica - $\mathrm{y}$ es en el interior de esa ambigüedad donde se ubican los usos que ciertos personajes hacen de las teorías en el proceso de su divulgación-. Más apropiado parece hablar de «recepción cultural», entendida como la elaboración, comunicación o recreación de los contenidos científicos teóricos por afuera de la literatura científica especializada (Fachliteratur). Por supuesto, la cuestión de cómo se expresa una teoría en la ciencia está ligada de manera inextricable a la pregunta sobre qué es una teoría, uno de los capítulos mayores de la filosofía y la retórica de la ciencia (ver, por ejemplo, Prelli; Gross).

En todo caso, uno de los formatos en la recepción de teorías científicas fueron las conferencias públicas pronunciadas por actores culturales prominentes. Vamos a concentrarnos en tres conferencias que introdujeron o difundieron entre nosotros tres teorías: la copernicana, la evolución y la relatividad. Estas tienen en común que su aceptación involucró conflictos severos antes de ingresar a la corriente principal de la ciencia, donde — al menos hasta ahora — han permanecido. Aunque en parte ya me ocupé de estas conferencias canónicas, espero que considerarlas de manera sinóptica permita echar alguna nueva luz sobre la utilización de la retórica científica como invocación a una socialmente compartida autoridad epistemológica de la ciencia y como inductora de significados clandestinos (intereses personales, programas de acción pública o sistemas de creencias). 


\section{Cerviño y la teoría heliocéntrica de Copérnico}

La conferencia con la que Pedro Cerviño, director de la Academia de Náutica del Consulado de Buenos Aires, inauguró a fines de enero de 1806 el período de exámenes públicos de esa escuela, constituyó una defensa de la importancia de la enseñanza de la astronomía en la formación de los pilotos. Copérnico aparece en ese texto como una de las figuras notables de esa ciencia (Cerviño 1806).

La alocución es una apología tanto de la astronomía como del propio Cerviño, quien encarnó desde la fundación de la academia, en 1799, el proyecto de una institución que proporcionase una extensa formación en matemáticas, frente a su competidor Juan Alsina, que aspiraba a hacer de la Academia una escuela de pilotaje de orientación práctica. El conflicto, que fue serio, se decidió durante el primer año de vida de la institución a favor de Cerviño. El ambicioso plan de estudios de este, apoyado por Belgrano, el promotor original de la Academia, estaba inspirado en las academias militares y navales de la España borbónica, punta de lanza de una modernización científica y técnica al servicio de un renovado instrumento imperial (es dable suponer que Cerviño haya estudiado en una de esas instituciones, quizás en la Academia militar de Barcelona) (Lafuente y Valverde:8-9, Vázquez Rivarola:37-42, Asúa 2010a:23-31).

Hacia fines del siglo XVIII, el relato histórico de la ciencia de los cielos era un molde cultural bien establecido en Francia, modelo de los españoles ilustrados. Había sido forjado, entre otros, por Giovanni Domenico Cassini en su trabajo «De l'origine et du progrès de l'astronomie et de son usage dans la géographie et la navigation» (1693), y se había consolidado en los cuatro volúmenes sobre astronomía antigua y moderna del astrónomo Jean Sylvain Bailly, más conocido por haber presidido el juramento de la cancha de pelota $(1775,1779)$. Tanto la Exposition du système du monde de Laplace (1796:199-312) como la Astronomie de Lalande (la primera edición es de 1764) incluían secciones substanciales dedicadas al origen y la historia de la astronomía (1771:97-232). El prefacio del tratado de Lalande, un savant emblemático de las Luces, es un elogio de dicha ciencia, con el que probablemente Cerviño estuvo familiarizado, ya que allí se cita el verso de Antoine-Marin Lemierre «le trident de Neptune est le sceptre du monde» que en versión castellana Cerviño usó como título de la conferencia del 25 de noviembre de 1799 con la que inauguró los cursos de la Academia de Náutica (Cerviño 1799, cf. Lalande:xx y Lemierre:405).

El discurso de 1806, que nos ocupa ahora, tuvo lugar frente a alumnos, quizás el virrey Sobremonte, el intendente, los miembros de la Junta del Consulado, su secretario Manuel Belgrano y otros notables (Besio Moreno:109). En él resume Cerviño la epopeya de la ciencia de Urania. Este ingeniero militar parte del conocimiento sobre el cielo de los egipcios, quienes lo habrían tomado de los caldeos, y concluye en una nota triunfal con el entonces relativamente reciente descubrimiento del planeta Urano por William Herschel en 1781. Cerviño ejemplifica la astronomía de la antigüedad griega con Aristarco, Eratóstenes e Hiparco, obviamente pasa por alto a Ptolomeo, y aterriza en «los árabes» y Alfonso X. De allí, saltamos al Renacimiento: «Apareció por fin Copérnico», quien "con un ánimo igual a su entendimiento chocó de lleno contra la opinión de todos los sabios de su tiempo [y] trató de persuadir que todo es ilusión, que el Sol y las Estrellas están inmóviles, que lo que se mueve es la masa del globo que habitamos» (Cerviño 1806:176). Sin embargo, el gran héroe de este tipo de narrativa ilustrada, cuya molde era el Discours préliminaire a la Encyclopédie de d'Alembert no era Copérnico, sino Newton. En efecto, en el discurso de Cerviño desfilan los protagonistas de la llamada «Revolución científica»: Galileo, Kepler, Huygens, y el «inmortal Newton». Cerviño sintetiza la ley de la inversa del cuadrado de la distancia y enumera los 
distintos fenómenos que Newton fue capaz de explicar en los Principia. Antes de culminar, no deja de referirse a los tres protagonistas del problema matemático de los tres cuerpos a mediados del siglo XVIII: Clairaut, d'Alembert y Euler. Esto era ciencia relativamente reciente.

Un largo párrafo del no muy extenso discurso está dedicado a enumerar el denso elenco de temas que Cerviño venía enseñando desde hacía cinco años, desde el álgebra hasta el «cálculo infinitesimal (...) cálculo que hará inmortales los nombres de Newton y Leibniz» (174). Luego seguían la dinámica y la hidrodinámica y, como coronación de este ambicioso esquema, «la astronomía, que eleva al hombre sobre sí mismo» y «la Teoría física de los cuerpos celestes y de las fuerzas recíprocas con que los Planetas alteran sus movimientos y hacen variar a los cometas el período de sus revoluciones» (o sea, los elementos de la mecánica celeste) (174). Conviene tener presente que una década atrás, en 1796, había aparecido la primera edición de la Exposición del sistema del mundo de Laplace, ya mencionado, y en 1799 el primero de los cinco tomos de su monumental Traité de mécanique céleste (1799-1825).

La aparición de Copérnico en el discurso de Cerviño se recorta sobre el fondo de las restricciones a la enseñanza del heliocentrismo. Si bien en 1757 fue eliminada del Index la cláusula que establecía la prohibición de enseñar el sistema heliocéntrico, se mantuvo la lista de cinco libros hasta entonces no permitidos (incluido, claro, el De revolutionibus), con lo cual en la primera década del siglo XIX la cuestión había quedado suspendida en una suerte de limbo (Finocchiaro:179-185). Desde hacía tiempo había copernicanos en el Río de la Plata, como lo fueron con toda probabilidad los ingenieros militares y navales que llegaron aquí para establecer la nueva frontera con Brasil como resultado del Tratado de 1777 entre España y Portugal. A cargo de la Tercera división de límites estaba el famoso naturalista ilustrado Félix de Azara, amigo y protector de Cerviño, quien integraba este grupo (Vázquez Rivarola:44). Pero la fortuna de Copérnico fue distinta en las aulas rioplatenses que entre estos hombres de teodolito y guerra. En la enseñanza, el sistema copernicano era expuesto como alternativa al de Ptolomeo y al de Tycho Brahe y no podía ser defendido sino hipotéticamente. Por caso, el jesuita Benito Riva, en el curso de filosofía de la naturaleza dictado en la universidad de Córdoba en la década de 1760, se inclinaba por el heliocentrismo, al afirmar que "Los Phisicos modernos quasi todos son Copernicanos». Esta es la única frase en castellano en el manuscrito latino, tomada literalmente de las Cartas eruditas del benedictino ilustrado Feijóo (310v; 299). Pero Riva no deja de subrayar la salvedad de que este sistema debía sostenerse como hipótesis («quod semper in hypothesi esse permissum») (314v-315r). Algo similar ocurrió durante el período franciscano en la universidad de Córdoba, y en los cursos y defensas de tesis en el Colegio de San Carlos y los estudios conventuales en Buenos Aires. El peso del problema queda de manifiesto en un breve tratado de Jorge Juan, el marino más importante de la España ilustrada, titulado Estado de la astronomía en Europa (3-15). El libro, una extendida historia apologética de la astronomía (algo así como la versión larga del discurso de Cerviño), se resuelve en una franca crítica de la necesidad de tener que acompañar la enseñanza de los tres sistemas del mundo con la refutación del copernicano y del newtonianismo (Juan:15). Lo de Cerviño es importante, porque constituyó una presentación pública del heliocentrismo — de hecho, la primera de que se tenga noticia- Pero no habría que perder de vista que la principal función de dicha referencia era la de integrar una legitimación retórica de un proyecto educativo tal como era el extenso plan de formación matemática ideado y llevado a cabo por aquel. 


\section{Sarmiento y la teoría de la evolución de Darwin}

El 19 de mayo de 1882, a tan solo a un mes de la muerte de Darwin, el Círculo Médico Argentino organizó un homenaje que atrajo un público que la optimista crónica oficial calculó en 4000 personas (Novaro:546). El acto tuvo lugar en el flamante Teatro El Nacional, inaugurado en febrero de ese año y situado en la calle Florida 146 (Zayas de Lima:25). Hablaron Sarmiento y Holmberg. La conferencia del primero, que merece la fama que la rodea, tiene dos partes (Sarmiento 1882). En la primera se celebra a Darwin y a la evolución; la segunda tiene que ver con lo que Sarmiento llama «la evolución del pensamiento» y es algo así como una cronología de la «civilización» (en el sentido sarmientino del término). Es de destacar que en la crucial mitad de este relato, que traza la larga parábola del mono a Edison, aparece como hito después de la creación de la imprenta en 1400, el siguiente (en bastardilla en la versión impresa de las obras completas): «Educación común universal para que todos puedan leer lo escrito. Cesa el Presbiterio de enseñar en las Escuelas de las Catedrales. LA INSTRUCCIÓN SE HACE LAICA» (el último enunciado en versalitas; 124). Este énfasis no es de sorprender. Darwin tuvo un lugar en las discusiones que rodearon la sanción de la ley de educación de 1884 en el ámbito de las medidas secularizadoras del primer gobierno de Roca (Asúa 2009). El autor del Origen de las especies, y en general el discurso científico del oficialismo, funcionaban como instrumento de legitimación (o fundamento, según se quiera mirar) de estas políticas. Pero la conferencia de Sarmiento alberga complejidades que no se limitan a la coyuntura laicista del ochenta, que por cierto no desdeña. Constituye un manifiesto de una fe evolucionista generalizada, y con esto, de una aspiración a integrar la circunstancia local en una cosmovisión universal. Dos énfasis del discurso son, si se quiere, el mismo: la apropiación de Darwin como actor de la escena argentina y la afirmación de que es en nuestro país donde se escriben grandes capítulos del libro de la naturaleza. El campo de lo que se conoce como «la ciencia de Sarmiento» puede organizarse en torno al dipolo conformado por el astrónomo Benjamin Gould y el naturalista Hermann Burmeister, que son Córdoba y Buenos Aires y son también los cielos y la tierra (Asúa 2010b:85-101; Montserrat). Hay otros dos nombres en la conferencia que expanden el panorama en direcciones divergentes. Louis Agassiz, el director del Museum of Comparative Zoology de Harvard (como a Gould, Sarmiento lo conocía de su estadía en Boston), constituía la contrapartida boreal de Burmeister, director del Museo Público de Buenos Aires y radicado en Buenos Aires por obra del sanjuanino. Ameghino, por su lado, era la generación de relevo, la de los argentinos Holmberg y P. Francisco Moreno. Sarmiento menciona 29 veces a Darwin, siete a Agassiz, y cinco a Burmeister, Gould y Ameghino. Los naturalistas franceses (d'Orbigny, Bravard, Bonpland) reciben apenas una mención cada uno. Darwin es, claro, el protagonista y estrella de primera magnitud de esta pléyade de «grandes nombres que figuran en los anales de la ciencia [y] se ligan a nuestra historia» (Sarmiento 1882:105). Sobre la base de los años que el creador de la evolución pasó en el cono sur de América, Sarmiento argumenta que su teoría nació «aquí mismo, en nuestros propios campos» (109), le hemos dado «fama a Darwin, con los fósiles y las crías argentinas» (110) y es por eso que «es uno de nuestros propios sabios» (106). Darwin es maestro de criadores, afirma, de tal modo que «hemos constituido una nueva especie, la "oveja argentífera", porque da plata y porque es argentina además» (110).

De resignada aunque legítima plata, no de oro, era el elenco que Sarmiento había podido reunir para sentar las bases de su Argentina científica y moderna, donde «el sabio Burmeister, el primer paleontólogo, (...) escribe desde nuestra patria la Historia de la Creación, mientras que el sabio 
Gould prepara la última edición de Los Cielos, corregida y aumentada considerablemente, desde nuestro Observatorio de Córdoba» (106). La Argentina se unía así «al progreso de la ciencia» (133), que había comenzado, dice Sarmiento, en el siglo XV, «el [siglo] libertador del género humano por la pólvora y por la prensa» (130). Darwin, Agassiz, Gould, Burmeister «siguen a nuestra vista ensanchando más y más aquellos límites» (126).

Veterano de la tinta fresca y curtido en todas las tribunas, Sarmiento alega carecer de autoridad para hablar de "ciencias naturales» ante los miembros de Círculo Médico y se hace fuerte en el punto de vista de «la vida pública», que ha sido, dice, su «provincia especial» (106). En un despliegue de familiaridad con los temas de la ciencia de su tiempo, menciona el descubrimiento del planeta Neptuno, el análisis espectral de las estrellas, la teoría microbiana de Pasteur y la antisepsia de Lister. En cuanto a sus ideas sobre la evolución de la vida en la Tierra, estas eran, para los estándares de ese momento, irremediablemente confusas. Sarmiento fue un evolucionista spenceriano convicto y confeso. En carta al Perito Moreno del 9 de abril de 1883, el sanjuanino distingue entre los «darwinistas» y su propio evolucionismo: «con Spencer me entiendo, porque andamos el mismo camino» (1883:322). La evolución de Sarmiento es la direccional de Spencer, no la de Darwin, la ordenada «de lo simple a lo compuesto, de lo embrionario a lo complejo, de la forma inacabada a la belleza acabada» (Sarmiento 1882:119). Por cierto, no hay una sola mención del Origen en todo el discurso. Sí del Viaje de un naturalista, que Sarmiento dice haber leído, como afirma también que conoció al Beagle y a su tripulación (ambos habrían coincidido en Chile). Sarmiento repite un largo fragmento de una de las «Seis conferencias para trabajadores» de Thomas Huxley (36) y cita también del Journey in Brazil de Agassiz y Elizabeth Cabot, su esposa (33, 42, 287, 383). Los ejemplos que da sobre evolución (los infaltables pinzones de las Galápagos y los eucaliptos de Australia) no dejan del todo claro que haya comprendido los mecanismos de selección natural. Sin embargo, sabe que Darwin atribuyó «la variación de las formas orgánicas a la selección natural de los tipos más vigorosos y adaptables al medio ambiente para la lucha por la existencia» (Sarmiento 1882:128).

Estas ambivalencias son entendibles (de hecho, el mismo Huxley nunca aceptó la selección natural). Lo que marca que Sarmiento prefería navegar por la brillante superficie discursiva de las cosas a sumergirse en las aguas profundas de las teorías, es la cuestión del catastrofismo, la idea de que la historia del planeta fue una serie sucesiva de cataclismos, seguidos de una renovación de las especies vivas. No es de extrañar, los dos sabios con los que había entablado contacto vivo y personal, Burmeister y Agassiz, eran catastrofistas. El Museo de Buenos Aires, dice Sarmiento en la conferencia, «es un verdadero cementerio de pasadas creaciones» (112) y en seguida precisa que el geólogo Elie de Beaumont ha contado «veinte y seis» de ellas (113). ¿No habrá una ley, se pregunta, que unifique «este desparramo de creaciones en millones de años, reemplazándose unas a otras, introduciendo más avanzadas formas bajo el mismo tipo, hasta aparecer el hombre» (115), que ha sido «contemporáneo de dos o tres creaciones de animales extintos»? (116). Cuando, siguiendo implícitamente a Cuvier, padre del catastrofismo, Sarmiento describe las sucesivas faunas de la cuenca de París descritas por el paleontólogo francés en 1812, lo hace en términos de una evolución que progresa en términos de complejidad: «el cachorro de elefante de hoy se parece al adulto de entonces [es decir, de un lecho inferior, más antiguo]» (112). Las alusiones catastrofistas de Sarmiento erupcionan también en Facundo, en el párrafo en que compara la llegada de Rosas al poder con la caída de un meteorito (Sarmiento 1938:50). Ahora bien, después de tanto 
escenario catastrófico, resulta que los geólogos, dice Sarmiento, «han llegado a convencerse de que las formas que han producido la extructura [sic] actual de la tierra, no son debidas a violentas convulsiones periódicas (...) sino a las fuerzas que operan continuamente» (Sarmiento 1882:131).

Pero entonces, ¿catastrofismo o uniformitarismo? ¿creaciones sucesivas o evolución gradual de las especies? ¿selección natural o transmisión de caracteres adquiridos? ¿Darwin o Spencer? Las respuestas a estas disyunciones excluyentes, cruciales para la ciencia, quizás no importaban demasiado para la lógica sarmientina, menos cercana al análisis que a la persuasión, si la misión era «llevar la teoría de Darwin, para explicar la influencia social que tales movimientos en las ideas ejercen en nuestra época» (119). Sí, y a la vez desparramar a los cuatro vientos el evolucionismo liberador de las tiranías de la mente, «aquí, en nuestro país, en la Pampa y en la Patagonia» (116), como «nuestro compatriota Benjamín Franklin» (132) quien, como afirma el epígrafe atribuido a Turgot, «arrebató al cielo el rayo y el cetro a los tiranos» [eripuit coelo fulmen sceptrumque tyrannis] (132; sobre la atribución, ver Anónimo, 1844).

\section{Lugones y el espacio de la relatividad}

El 14 de agosto de 1920 Leopoldo Lugones dio una conferencia invitado por el Centro de Estudiantes de la Facultad de Ciencias Exactas, Físicas y Naturales de la Universidad de Buenos Aires (que básicamente era la carrera de ingeniería), la que fue publicada un año más tarde como $\mathrm{El}$ tamaño del espacio. Ensayo de psicología matemática (1921). Allí Lugones desestima la idea de que sólo son válidos los conceptos que pueden ser intuidos (es decir, visualizados por la imaginación) y defiende que cualquier noción geométrica, en particular la de espacio, debe ser considerada en términos puramente racionales. Lugones se detiene en la geometría no-euclidiana de Riemann y en el espacio tetra-dimensional de Minkowski, instrumentos matemáticos íntimamente asociados a la relatividad general y un ejemplo de geometría no intuitiva. En la reseña de la publicación de la conferencia de Lugones, La Nación comentaba: «Lugones llega a la conclusión del Universo finito y, por ende, mensurable. Al poco tiempo, una crónica telegráfica de Berlín, publicada por este diario, dio cuenta de una conferencia en que el propio Einstein formulaba esta teoría» (La Nación 1921). O sea, para el cronista, Lugones se había anticipado a Einstein. ¿Qué pensaba sobre esto el propio Einstein? En su visita de marzo-abril de 1925 a Buenos Aires el físico fue entrevistado por un periodista de El Hogar, Eggers Lecour, que le preguntó: «¿Sabe (...) que Leopoldo Lugones ha declarado que el concepto de la finitud del espacio fue ya sostenido por él, varios años atrás, en su famosa conferencia de la Facultad de Ingeniería?». «Lo sé», responde Einstein, «pero las ideas del Sr. Lugones sobre este punto me parecen un poco...». «¿Relativas?», le sugiere el periodista. «iNo, nebulosas! —sonríe [Einstein] contento de haber hallado la palabra» (Eggers-Lecour).

En efecto, El tamaño del espacio es una mezcla de ideas sugestivas, confusas nociones matemáticas y gran cantidad de datos científicos empíricos, todo ello amalgamado en un estilo exuberante y declamatorio. Lugones abrevó de la literatura de difusión francesa, como la Revue scientifique (algo así como La Recherche de los años veinte), los libros de Jean Perrin y probablemente Poincaré. Que Lugones aspiraba a legitimarse en el propio terreno de la ciencia es evidente si se tiene en cuenta la mímesis aluvial de vocabulario matemático y físico que utiliza, por ejemplo: las transformaciones de grupo de Lie (Lugones 1921:17), las ecuaciones de Maxwell (19), la definición de geometría de Klein (20), «las experiencias de Fizeau, de Michelson y Morley, de Lorentz, de Eötvös, de Mach, de Einstein (...)» (21), los «experimentos geodésicos» y las «famosas «disquisiciones» de 
Gauss» (21), los cuaterniones de Hamilton (21), alguna hipótesis sobre el éter de Kelvin (35) y así. La voz de Lugones es aquí la impostada de un profesor de ciencias que despliega sus ínfulas en un martilleo de sentencias oraculares.

El tamaño del espacio está organizado sobre una antítesis subyacente que vuelve, una y otra vez, a emerger en nuevas formas. Se trata de la oposición entre intuición y razón plasmada como la contraposición entre espacio infinito y espacio finito indefinido. Las dos oposiciones, razón-intuición y espacio finito-espacio infinito, son manifestaciones de una tercera, más profunda: la que enfrenta el dogma religioso con la libertad. Asociado a la primera de estas contraposiciones hay un motivo alegórico: el de Prometeo encadenado que finalmente se libera. El titán, personificación de la razón matemática abstracta, nos permitió, al decir de Lugones, escapar de la esclavitud de los sentidos (20). «También el número, destacada invención, adquirí para ellos» le hace decir Esquilo a su Prometeo encadenado (1. 460), que Lugones menciona sin citar. Esta alegoría no tiene una mera función decorativa, sino aquella que Lugones había señalado en su propio Prometeo y que correspondería a todo relato mítico de orígenes: el de contar una evidencia en un lenguaje poético o figurativo, «admitiendo que existe en dichas ficciones alguna verdad general» (Lugones 1910:67).

En su recorrido por la enciclopedia total del conocimiento, desde las matemáticas hasta la teología, la conferencia de Lugones genera una sensación de autoridad intelectual aplastante a la que nada escapa. Este efecto está mediado por la utilización de adjetivos de sentido análogo, pero pertenecientes a distintos campos del saber. El escamoteo conceptual de Lugones se disuelve en la fascinación de una prosa que ablanda las duras aristas de los términos y conceptos de las ciencias exactas y los metamorfosea y combina en una rapsodia insinuante, hipnótica y, desde el punto de vista de la racionalidad científica, vacía del sentido que promete y no entrega.

Ya ha sido señalado que el "Ensayo de cosmogonía en diez lecciones», al final de Las fuerzas extrañas, es un relato cosmogónico en clave teosófica que aspira a competir con la cosmología de Laplace como explicación del origen del universo (Lugones 1906:201-278, Hewitt y Hall). Los temas centrales de El tamaño del espacio habían aparecido catorce años antes en este relato y hay hilos de continuidad entre el «Ensayo» y la conferencia de 1920. Uno de ellos es la noción de «éter». En la conferencia, Lugones concibe al éter como existiendo «en un estado de altísima tensión», lo que sería compatible "con su energía y su inercia eléctrica» (Lugones 1921:39). Esta caracterización, que en sí es apenas inteligible, está sin embargo en línea con la cosmogonía del «Ensayo» donde se afirma que «la primera manifestación del éter es, en efecto, electricidad» (Lugones 1906:212) y que «la materia es (...) electricidad neutra cuya tensión se ha transformado en gravedad» (239). En relación con la luz, Lugones afirma en la conferencia que «es invisible per se: su visibilidad resulta un fenómeno de transformación sensible, por el cual únicamente podemos definirla» (1921:25, énfasis en el original). Paralelamente, en el «Ensayo» Lugones había proclamado que, dado que la luz es la forma más elevada de energía pura, la misma equivale a las tinieblas, pues estas, según los antiguos, serían «luz absoluta» (1906:208-209) Este es el tipo de especulación que ocupa la tercera parte del primer volumen de The Secret Doctrine de Madame Blavatsky (1888 I:477-676; sobre la teosofía en Buenos Aires y en los relatos de Lugones, ver Quereilhac:125-159 y 203-235; Gasparini dejó a Lugones en el umbral de su inevitable trabajo sobre fantasía científica, 2012:40-42).

El tamaño del espacio plantea que alguna vez existió una «edad de Newton», caracterizada por el «espacio intuitivo» (infinito), por la creencia en la «materia ponderable o cuerpo físico del 
universo» y por la atracción gravitatoria. Esta época habría sido superada por la «era de Einstein», en la que las matemáticas revelan el espacio finito, la materia ponderable es reemplazada por el electromagnetismo y la atracción es sustituida por la luz (Lugones 1921:18-19). Todo esto admite ser leído como una historia un tanto imaginativa de la física. Pero lo que su autor está aquí codificando es una cosmogonía «espiritualista». En la conferencia de 1920 el espacio es concebido como un cuerpo que, suponemos que en un futuro indeterminado, «carecerá de peso, al ser solamente enérgico", pues él es "la suprema energía» de la cual surge "toda manifestación enérgica apreciable por nuestros medios». A la larga, la energía se agotará por el rozamiento, de tal modo que «reabsorbiéndose en un equilibrio incondicionado y negativo, pasará a otro estado en el espacio de Einstein: la superficie de curvatura constante variable» (37-38). La noción de energía propia de los físicos se fusiona insensiblemente con la «energía pura» de una sabiduría hermética y el período culmina con (o mejor dicho desciende hacia) la disolución del cosmos y su reaparición «en el espacio de Einstein» (38).

El tamaño del espacio, la conferencia que fue aplaudida por un público entusiasta de estudiantes de ingeniería y matemáticas, fue no sólo la evocación de algunas nociones de Einstein sino y fundamentalmente, una exposición teosófica. Esta fue la segunda oportunidad en que Lugones pulsó este registro. En el «Ensayo» de 1906 lo hizo con la excusa apenas velada de la ficción, en 1920 eligió una tribuna científico-profesional. El análisis de los contenidos de ambos textos, que aquí insinuamos y que desarrollamos con más detenimiento en (2006), demuestra su continuidad: Lugones fue consistente consigo mismo. Leída desde el punto de vista de la cosmogonía de 1906, la conferencia se revela como una reelaboración en la que se integran los últimos datos de la ciencia positiva a una matriz retórica ya constituida. La cosmología de Einstein es absorbida, sincréticamente, en la doctrina teosófica de la evolución cósmica del «Ensayo». La incandescencia verbal de Lugones funde los registros de modo tal que las connotaciones esotéricas de la conferencia resultan sólo aparentes para oídos que hubieran prestado atención al comunicador de la enseñanza hasta entonces guardada, que «en la soledad inspiradora de las noches andinas» (Lugones 1906:204) escancia la doctrina secreta del universo. Es para esos oídos que la enrarecida urdimbre de El tamaño del espacio adquiere su real significado.

\section{Conclusión}

En los casos que acabamos de discutir y que conciernen tres teorías troncales de la ciencia moderna, la exposición conceptual de estas estuvo asociada con (o al servicio de) intereses particulares, proyectos políticos o sistemas de ideas legitimados mediante la autoridad social de la ciencia. Este proceso se da, por ejemplo, mediante la apropiación calculada del vocabulario científico, mecanismos de deslizamiento y conjugación de significados o entusiasmos metafóricos. Los silencios, inconsecuencias y contradicciones del discurso que mimetiza el lenguaje de la ciencia, son otras tantas bocas de acceso a las regiones sumergidas que flotan ambiguamente entre la manifestación y el ocultamiento. La conferencia fue un formato efectivo en la difusión de teorías científicas hacia un público ávido de celebraciones colectivas de la ciencia y que, en algún caso, era cómplice voluntario de la ambivalencia (algunos dirían la impostura). Las complejidades que análisis como estos abren respecto de la recepción cultural de teorías y el papel de sus voceros no son de desestimar. 


\section{Notas}

1 Traté la conferencia de Cerviño en «"Los phisicos modernos quasi todos son copernicanos". Copernicanism and its Discontents in Colonial Río de la Plata» Journal of the History of Astronomy 48 (2) 2017, 1-20. La conferencia de Sarmiento fue ubicada

en contexto en (2004b:138-144). Con relación a la de Lugones, la consideré en relación con la recepción cultural de la relatividad en Buenos Aires (2006:240-261); dicha conferencia fue traducida al inglés (Asúa y Hurtado de Mendoza).

\section{Bibliografía}

Agassiz, Louis and Elizabeth [Cabot] Agassiz (1868). A Journey in Brazil. Boston: Tickner and Fields.

[Anónimo] (1844). «Franklin's Celebrated Line - “Eripuit Coelo Fulmen, etc.”». The United States Magazines and Democratic Review 15(78), 625-626.

Asúa, Miguel de (2004a). «Paradigma», en Francis Korn y Miguel de Asúa, editores. Errores eruditos y otras consideraciones. Buenos Aires: Instituto de Investigaciones Sociales, ANCBA, 95-102.

(2004b). Ciencia y literatura. Un relato histórico. Buenos Aires: Eudeba.

- (2006). «Relatividad y literatura en Argentina», en Miguel de Asúa y Hurtado de Mendoza, Imágenes de Einstein. Relatividad y cultura en el mundo y en la Argentina. Buenos Aires: Eudeba, 239-287. (Ed.) (2009). «Abogados, médicos y monos. Darwin y los católicos en la Argentina del siglo XIX». Los significados de Darwin. Buenos Aires: Academia Nacional de Ciencias de Buenos Aires, 39-51. (2010a). La ciencia de Mayo. La cultura científica en el Río de la Plata, 1800-1820. Buenos Aires: Fondo de Cultura Económica. (2010b). Una Gloria silenciosa. Dos siglos de ciencia en Argentina. Buenos Aires: Zorzal.

Asúa, Miguel de y Diego Hurtado de Mendoza (Trads.) (2005). «The Size of Space (An Essay on Mathematical Psychology). Leopoldo Lugones». Science in Context 18(2), 317-336.

Bailly, Jean-Sylvain (1775). Histoire de la astronomie ancienne, depuis son origine jusqu'à l'établissement de l'école d'Alexandrie. París: Frères Debure.

(1779). Histoire de la astronomie moderne,depuis la fondation de l'école d'Alexandrie, jusqu'à l'époque de M.D.CC.XXX, 3 vols. París: Chez De Bure.

Besio Moreno, Nicolás (1995). Las fundaciones matemáticas de Belgrano. Buenos Aires: Instituto Nacional Belgraniano.

Blavatsky, Helena Petrovna (1888). The Secret Doctrine. The Synthesis of Science, Religion and Philosophy, 2 vols. Londres: Theosophical Publishing Society.

Cassini, Giovanni Domenico (1693). «De l'origine et du progres de l'astronomie et de son usage dans la géographie et la navigation». Recueil d'observations faites en plusieurs voyages par ordre de sa Majesté pour perfectionner l'astronomie et la géographie. Avec divers traitez astronomiques par messieurs de l'Académie Royale des Sciences. París: Imprimerie Royale.

Cerviño, Pedro (1799). «El tridente de Neptuno es el cetro del mundo», en Nicolás Besio Moreno. Las fundaciones matemáticas de Belgrano. Buenos Aires: Instituto Nacional Belgraniano, 1995, 159-173. (1806). «Prolusión académica. Discurso en que se procura que para ser buen piloto es necesaria la astronomía», en Nicolás Besio Moreno. Las fundaciones matemáticas de Belgrano. Buenos Aires: Instituto Nacional Belgraniano, 1995, 173-178.

D’Alembert, Jean Le Rond (1763). Discours préliminaire de l'Encyclopédie. F. Picavet, editor. París: Armand Colin, 1929.

Eggers-Lecour, Conrado (1925). «Einstein, la inteligencia más madura y el espíritu más joven del siglo». El Hogar 21(809), 7 y 64 . 
Esquilo (2000). «Prometeo encadenado». Tragedias. Madrid: Gredos, 269-312. Traducción de Bernardo Perea Morales.

Feijóo, Bernardo (1753). «Progresos del Sistema Filosófico de Newton, en que es incluido el Astronómico de Copérnico». Cartas eruditas y curiosas. Madrid: Joachin Ibarra, 1770, IV:294-08.

Finocchiaro, Maurice A. (2010). Defending Copernicus and Galileo. Critical Reasoning in the Two Affairs. Dordrecht: Springer.

Gasparini, Sandra (2012). Espectros de la ciencia. Fantasías científicas de la Argentina del siglo XIX. Buenos Aires: Santiago Arcos.

Gross, Alan G. (1990). The Rhetoric of Science. Cambridge (Massachusetts): Harvard University Press.

Hewitt, Sandra y Nancy Abraham Hall (1984). «Leopoldo Lugones y H. P. Blavatsky. Teosofía en el “Ensayo cosmogónico en diez lecciones"». Revista de Estudios Hispánicos 18(3), 335-343.

Holub, Robert C. (2003). Reception Theory. Abingdon, Oxon.: Routledge.

Huxley, Thomas (1863). «The Past Condition of Inorganic Nature». Twelve Lectures and Essays. Londres: Watts, 1908, 27-37.

Jauss, Hans R. (1969). «Paradigmawechsel und Literaturwissenschaft». Linguistische Berichte 3, 44-56. Juan, Jorge (1774). Estado de la astronomía en Europa y juicio de los fundamentos sobre que se erigieron los Systemas del Mundo, para que sirva de guía al método en que debe recibirlos la Nación, sin riesgo de su opinión y de su religiosidad. Madrid: Imprenta Real de la Gaceta.

Kuhn, Thomas S. (1962). The Structure of Scientific Revolutions. Chicago: University of Chicago Press.

Lafuente, Antonio y Nuria Valverde (2003). Los mundos de la ciencia en la Ilustración Española. Madrid: Fundación española para la ciencia y la tecnología.

Lalande, Jerôme (1771). Astronomie, 2a ed., 3 vols. París: chez la veuve Desaint.

[La Nación (Buenos Aires)] (1921, 13 de febrero). «"El tamaño del espacio” por Leopoldo Lugones», La Nación, sección 2, 6 .

Laplace, Pierre Simon de (1796). Exposition du système du monde, 2 vols. París: Imprimerie du Cercle-Social. ---. (1799-1825). Traité de mécanique céleste, 5 vols. París: J. B. M. Duprat (1-3), Courcier (4), Bachelier (5). Lemierre, Antoine-Marin (1755). «Le Commerce». Élite des poésies décentes. Lyon: Perisse, 1772, II:402-405. Lugones, Leopoldo (1906). «Ensayo de cosmogonía en diez lecciones». Las fuerzas extrañas. Buenos Aires: Arnoldo Moen y Hermano, 201-278.

(1910). Prometeo (un proscripto del sol). Buenos Aires: Otero \& Co.

(1921). El tamaño del espacio. Un ensayo de psicología matemática. Buenos Aires: El Ateneo.

Montserrat, Marcelo (1988). «Sarmiento, propulsor de la ciencia». Ciencia e Investigación 42(5), 277-283.

Nauta, Ruurd R. (1994). «Historicizing Reading: The Aesthetics of Reception and Horace's "Soracte Ode"», en Irene J. F. de Jong y J. P. Sullivan, editores. Modern Critical Theory and Classical Literature. Leiden: Brill, 207-230.

Novaro, Bartolomé (1882). «Informe sobre la marcha del “Círculo Médico” hecho por su presidente en la sesión del 29 de junio». Anales del Círculo Médico Argentino, 5, $1^{\circ}$ de agosto, 543-547.

Prelli, Lawrence J. (1989). A Rhetoric of Science: Inventing Scientific Discourse. Columbia, S. C.: University of South Carolina Press.

Quereilhac, Soledad (2016). Cuando la ciencia despertaba fantasías. Prensa literatura y ocultismo en la Argentina de entresiglos. Buenos Aires: Siglo XXI.

Riva, Benito (1760?). [Cursus physicae], De mundo et caelo, ff. 306v-319r, Ms. en Buenos Aires, Fondo Antiguo de la Compañía de Jesús en Argentina. 
Sarmiento, Domingo F. (1845). Facundo. Alberto Palcos, edición crítica. La Plata: Universidad Nacional de La Plata, 1938.

(1882). «Darwin», en Augusto Belín Sarmiento y Luis Montt, editores. Obras de D. F. Sarmiento, vol. 22. Buenos Aires: Librería La Facultad, 1913, 104-133.

«Carta a Francisco P. Moreno, Buenos Aires, 9 de abril de 1883», en Augusto Belín Sarmiento, editor. Obras de D. F. Sarmiento, vol. 36. Buenos Aires: Imprenta Mariano Moreno, 1900, 322-326.

Turner, Henry S. (2010). «Lessons from Literature to the Historian of Science (and Vice Versa). Reflections on “Form"». Isis 101, 578-588.

Vázquez Rivarola, Horacio Guillermo (2009). Cerviño, hijo de Galicia, padre de Argentina. Vigo: Grupo de Comunicación de Galicia en el Mundo.

Zayas de Lima, Perla (1999). «Espectáculos teatrales y para-teatrales en el Buenos Aires del siglo XIX», en Liliana Barela, editora. Teatro y Literatura. Buenos Aires: Instituto Histórico de la Ciudad de Buenos Aires, 9-32. 